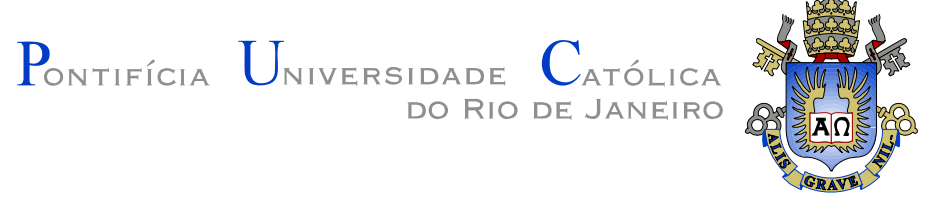

Maiquel dos Santos Canabarro

\title{
Análise e Síntese de Refletores Circularmente Simétricos pelos Métodos da Ótica Física e Correntes de Franja
}

Dissertação de Mestrado

Dissertação apresentada ao Programa de PósGraduação em Engenharia Elétrica da PUC-Rio como requisito parcial para obtenção do título de Mestre em Engenharia Elétrica da PUC-Rio.

Orientador: José Ricardo Bergmann Co-orientador: Flávio José Vieira Hasselmann

Rio de Janeiro

Maio de 2009 


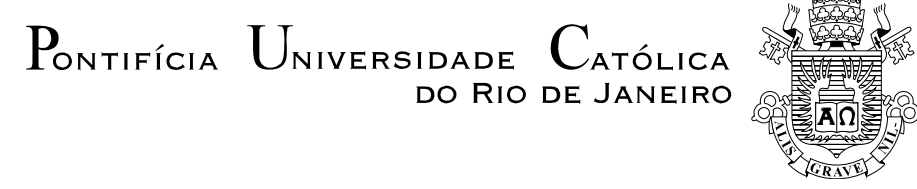

Maiquel dos Santos Canabarro

\title{
Análise e Síntese de Refletores Circularmente Simétricos pelos Métodos da Ótica Física e Correntes de Franja
}

\begin{abstract}
Dissertação apresentada como requisito parcial para obtenção do título de Mestre pelo Programa de PósGraduação em Engenharia Elétrica da PUC-Rio. Aprovada pela Comissão Examinadora abaixo assinada.
\end{abstract}

Dr. José Ricardo Bergmann

Orientador

Centro de Estudos em Telecomunicações - PUC-Rio

Dr. Flávio José Vieira Hasselmann

Co-Orientador

Centro de Estudos em Telecomunicações - PUC-Rio

Dr. Luiz Costa da Silva

Centro de Estudos em Telecomunicações - PUC-Rio

Dr. Fernando José da Silva Moreira Universidade Federal de Minas Gerais - UFMG

Dr. José Eugênio Leal

Coordenador(a) Setorial do Centro Técnico Científico - PUC-Rio

Rio de Janeiro, 29 de maio de 2009 
Todos os direitos reservados. É proibida a reprodução total ou parcial do trabalho sem autorização da universidade, do autor e do orientador.

\section{Maiquel dos Santos Canabarro}

Graduou-se em Engenharia Elétrica, em julho de 2006, na Universidade Federal de Santa Maria. Em agosto do mesmo ano, iniciou no Centro de Estudos em Telecomunicações da Pontifícia Universidade Católica do Rio de Janeiro seu mestrado na Área de Eletromagnetismo Aplicado.

Ficha Catalográfica

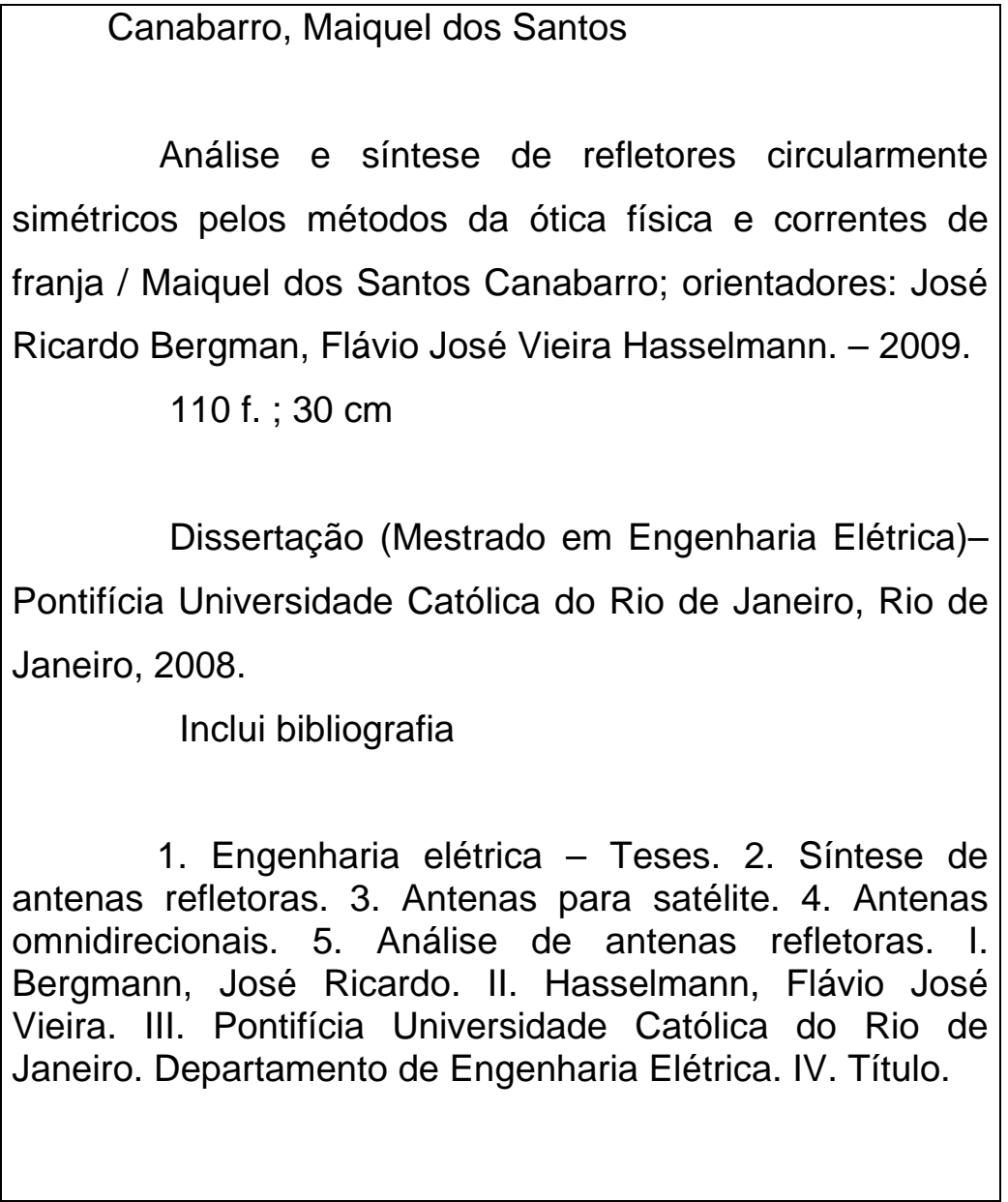

CDD: 621.3 
A minha família, em especial aos meus pais Miriam e Verci, e a minha esposa Camila. 


\section{Agradecimentos}

Ao meu Orientador Dr. José Ricardo Bergmann pelas incansáveis orientações, pela paciência, e principalmente pelos ensinamentos ao longo de nosso trabalho e por acreditar em mim.

Ao meu Co-Orientador Dr. Flávio José Vieira Hasselmann pelas orientações e paciência durantes as etapas desse trabalho.

Ao Dr. Nelson Jorge Schuch por me mostrar a importância das nossas liberdades.

A minha Família: meus irmãos Rita e Moisés, os meus tios Ana e Wilson e em especial aos meus pais Miriam e Verci pelo carinho e por proporcionar minha educação.

A meus sogros Dalva e Aldenir, aos meus cunhados Gilson e Francisco pelo apoio e carinho.

A minha esposa, Camila, por acreditar no projeto de vida que juntos estamos construindo dia a dia, pelo amor, pelo carinho, pelos incansáveis incentivos ao longo do percurso deste trabalho.

Ao Dr. Cássio Gongalvez do Rego, UFMG, pelas informações teóricas sobre sua Tese.

Aos colegas do CETUC, Sandro, Djeisson, Ramariz, Marco Aurélio, Fabrício, 
André, Rafael pela amizade e companheirismo no dia a dia do trabalho.

Aos amigos Robinson, Fábio e João Paulo pela acolhida no momento em que cheguei aqui no Rio.

Aos funcionários da PUC-Rio, em especial a as Anas Sergio, a Célia e a Alcina.

A CAPES, ao CNPq e à PUC-Rio, pelos auxílios concedidos, sem os quais esse trabalho não poderia ter sido realizado.

A Deus, por nos mostrar a energia do Universo todos os dias, e assim nos ensinado a viver a vida com sabedoria. 


\section{Resumo}

Canabarro, Maiquel dos Santos; Bergmann, José Ricardo; Hasselmann, Flávio José Vieira. Análise e Síntese de Refletores Circularmente Simétricos pelos Métodos da Ótica Física e Correntes de Franja . Rio de Janeiro, 2009. 110p. Dissertação de Mestrado - Departamento de Engenharia Elétrica, Pontifícia Universidade Católica do Rio de Janeiro.

O projeto de antenas refletoras para a geração de feixes modelados envolve a construção de algoritmos baseados em métodos de análise eletromagnética associados a técnica de otimização. Em geral, estes algoritmos demandam considerável tempo de processamento computacional, impondo limites para sua utilização intensiva na exploração de diferentes conjuntos de especificações e na busca de soluções mais compactas. Neste trabalho, será considerada a síntese de antenas refletoras circularmente simétricas para a geração de feixes modelados circularmente simétricos. Em função das características de simetria da antena, simplificações serão introduzidas na formulação do problema, reduzindo substancialmente o tempo de processamento e viabilizando sua utilização intensiva. Para a análise eletromagnética da antena refletora serão consideradas as aproximações da Ótica Física (PO) e das correntes de franja (CF). Considerando alimentadores radiando um campo com dependência azimutal $\mathrm{n}=1$, o algoritmo foi adaptado e aplicado no modelamento de antena refletora para atender as especificações do CBERS (China-Brazil Earth Resources Satellite). Para alimentadores com dependência azimutal $n=0$, foram considerados sistemas de antenas omnidirecionais de um e dois refletores para a geração de diagramas modelados no plano vertical. Para estes dois tipo de sistema, o algoritmo de modelamento foi utilizado para maximizar o diagrama em direção e para produzir diagramas com dependência do tipo cossecante ao quadrado no plano vertical. A validação da técnica de análise eletromagnética desenvolvida foi obtida comparando os resultados com os gerados via Métodos dos Momentos.

\section{Palavras-chave}

síntese de antenas refletoras; antenas para satélite; antenas omnidirecionais; análise de antenas refletoras 


\section{Abstract}

Canabarro, Maiquel dos Santos; Bergmann, José Ricardo (advisor); Hasselmann, Flávio José Vieira (advidor). Synthesis and Analysis of Circularly Symmetric Reflectors via Physical Optics and Fringe Wave Currents. Rio de Janeiro, 2009. 110p. MSc. Dissertation - Departamento de Engenharia Elétrica, Pontifícia Universidade Católica do Rio de Janeiro.

The usual algorithms employed in the design of shaped reflector antennas for satellite applications embed an electromagnetic analysis method in an optimization technique. In general, these algorithms demand considerable computation time, limiting its intensive use in the study to explore different sets of specifications and to search for more compact geometries. In this work, it is considered the particular case of the shaping of circularly symmetric reflectors antennas for the generation of circularly symmetric radiation patterns. The use of the reflector surface properties simplifies the formulation of the electromagnetic scattering and substantially reduces the computer time involved in the optimization iteration. For the electromagnetic scattering, the algorithm employed here considers the approximations given by the Physical Optics and Fringe Currents methods. Firstly, the shaping algorithm was applied in the design of a single reflector antenna to comply with the specifications of the China-Brazil Earth Resources Satellite (CBERS). For this case, the feed radiation pattern was represented by model with azimuthal dependence $n=1$. As a second case, the shaping algorithm was applied in the design of single and dual reflector omnidirectional antennas. Theses antennas were feed by a TEM coaxial horn with azimuthal dependence $n=1$. For these two type of antenna configurations, the modelling algorithm was used to maximize the diagram in one direction or to produce radiation patterns with dependence of the type cosecant to the square in the vertical plan. A Methods of Moments electromagnetic analysis was used to validate the scattering fields yielded by the PO+Fringe currents approximations.

\section{Keywords}

Synthesis reflectors antennas; satellite antennas; omni antennas; Analysis reflectors antennas 


\section{Sumário}

1 Introdução

2 Análise de refletores circularmente simétricos

alimentados por diagrama $\mathrm{n}=1$

2.1. Introdução 19

2.2. Modelo de alimentador para $n=1 \quad 19$

2.3. Refletores circularmente simétricos 22

2.4. Determinação do campo distante usando

Aproximações da Ótica Física (PO) 24

2.4.1. Formulação para um único refletor

circularmente simétrico 25

2.4.2. Determinação das correntes equivalentes

de borda e correntes de franja 28

2.5. Validação do algoritmo de análise 36

3 Síntese de refletores circularmente simétricos

alimentados por diagrama com dependência azimutal $n=1$

3.1 Antenas para cobertura uniforme em satélites

de baixa órbita: Caso CBERS 39

3.2 Técnica de otimização de refletores 41

3.3 Solução inicial via síntese ótica 44

3.4 Modelamento de refletores para cobertura do CBERS 47

4 Análise de refletores circularmente simétricos

alimentados por diagramas com dependência azimutal $n=0 \quad 59$

4.1 Introdução $\quad 59$

4.2 Modelos de alimentadores com dependência azimutal $n=0 \quad 60$

4.3 Formulação para um único refletor circularmente simétrico 63

4.4 Formulação para duplos refletores circularmente simétricos 65

4.5 Correntes de Franja aplicadas na formulação para refletores 
alimentados por alimentadores com dependência azimutal $n=0$

4.6 Comparação de resultados de análise de casos

descritos na literatura

4.6.1 Comparação de resultados de análise de casos com um único refletor

4.6.2 Comparação de resultados de análise de antenas com duplos refletores

5 Síntese de refletores circularmente simétricos alimentados por diagrama com dependência azimutal $n=0$

5.1 Introdução

5.2 Caso de antenas refletoras omnidirecional com um único refletor

5.2.1 Antenas refletoras omnidirecionais com diagrama

do tipo cossecante ao quadrado no plano vertical

5.3 Caso de antenas refletoras omnidirecional de duplos refletores

6 Considerações Finais

Referências Bibliográficas

Apêndice A Transformação das integrais duplas em lineares 


\section{Lista de figuras}

Figura 2.1 - Refletor circularmente simétrico 22

Figura 2.2 - Orientação do vetor unitário normal a superfície. 24

Figura 2.3 - Ângulos e vetores utilizados nas equações das

correntes e do coeficiente de difração por uma cunha curva. 30

Figura 2.4 - Ângulos e vetores utilizados nas equações das correntes

e dos coeficientes de difração por borda de uma antena refletora.

Figura 2.5 - Sub-refletor hiperbólico com excentricidade

igual a, $\varepsilon=2$, implementado no caso. 37

Figura 2.6 - Diagrama de campo espalhado para a polarização 37

Figura 2.7 - Diagrama de espalhamento para o polarização Principal 38

Figura 3.1 - Características da orbita do CBERS. 40

Figura 3.2 - Especificação para o diagrama de radiação para o CBERS. 41

Figura 3.3 - Limites mínimo e máximo das especificação

para o diagrama de radiação para o CBERS com faixa de $3 \mathrm{~dB}$.

Figura 3.4 - Relação de $\theta\left(\theta^{\prime}\right)$ e o principio da conservação de

energia no interior do tudo de raios. 45

Figura 3.5 - Geratrizes dos refletores sintetizados pela GO para 50 e 60 cm. 46

Figura 3.6 - Diagrama de radiação do refletor sintetizado pela GO. 47

Figura 3.7 - Diagramas de radiação sintetizados pela PO+CF com

5 e 7 termos na Serie de Fourier. 49

Figura 3.8 - Diagramas de radiação sintetizados pela PO+CF

com 8,12 e 16 termos na Série de Fourier.

Figura 3.9 - Geratriz dos refletores sintetizados com 5 e 7

termos na Série de Fourier.

Figura 3.10 - Geratriz dos refletores sintetizados com mais 8 termos

na Série de Fourier.

Figura 3.11 - Curvas limites, $\mathrm{G}_{\mathrm{MAX}}$ e $\mathrm{G}_{\mathrm{MIN}}$, para diagrama

de radiação de cobertura do projeto Cbers.

Figura 3.12 - Diagramas de radiação sintetizados pela PO+CF

com 7 e 8 termos na Série de Fourier. 
Figura 3.13 - Diagramas de radiação sintetizados pela PO+CF

com 10, 12 e 16 termos na Série de Fourier.

Figura 3.14 - Geratriz dos refletores sintetizados com 7 e 8 termos

na Série de Fourier.

Figura 3.15 - Geratriz dos refletores sintetizados com 10,12 e

16 termos na Série de Fourier.

Figura 3.16 - Destaque no diagrama de radiação do sintetizado

com 7 termos na Série de Fourier.

Figura 3.17 - Diagramas de radiação sintetizados pela PO+CF

com diferentes números de termos na Série de Fourier.

Figura 3.18 - Geratriz dos refletores sintetizados com 8 a 16 termos na Série de Fourier.

Figura 3.19 - Diagramas de radiação sintetizados pela PO+CF

com 12,14 e 16 termos na Série de Fourier para o refletor de 50 cm.

Figura 3.20 - Destaque no diagrama de radiação do sintetizado

com 12,14 116 termos na Série de Fourier.

Figura 3.21 - Diagramas de radiação sintetizados pela PO+CF

com 14 termos na Série de Fourier e aplicação dos pesos Wm.

Figura 3.22 - Geratriz dos refletores sintetizados com 14 termos na Série de Fourier.

Figura 4.1 - Dependência axial dos refletores e alimentador.

Figura 4.2 - Corneta coaxial corrugada utilizada na alimentação

de antenas refletoras com radiação omnidirecional.

61

Figura 4.3 - Diagrama de radiação da corneta coaxial corrugada.

Figura 4.4 - Disposição dos vetores usados no cálculo das

correntes induzidas sobre as superfícies dos refletores da antena.

Figura 4.5 - Comparação entre os diagrama de radiação do alimentador medido com o gerado por um disco com raios internos e externos iguais $0.43 \lambda$ e $0.93 \lambda$, respectivamente.

Figura 4.6 - Configuração da antena refletora de um único refletor com radiação ominidirecional.

Figura 4.7 - Diagramas de radiação de uma antena refletora ominidirecional analisada pelos métodos PO, PO+CF e modelo 
aproximado comprando com o MoM.

Figura 4.8 - Diagramas de radiação de uma antena refletora

ominidirecional analisada pelos métodos $\mathrm{PO}, \mathrm{PO}+\mathrm{CF}$ alimentados pela

expansão de ondas esféricas comparado com o MoM.

Figura 4.9 - Antena refletora de duplo refletora configurada com

um subrefletor parabólico e um principal dado por uma cônica.

Figura 4.10 - Diagramas de radiação da antena refletora omnidirecional com dois refletores analisadas pelo $\mathrm{PO}, \mathrm{PO}+\mathrm{CF}$ e MoM.

Figura 4.11 - Diagramas de radiação da antena refletora PACO

analisadas pelo $\mathrm{PO}, \mathrm{PO}+\mathrm{CF}$, alimentada pela expansão de ondas esféricas, e MoM.

Figura 4.12 - Antena refletora de duplo refletores configurada OADE.

Figura 4.13 - Diagrama de radiação da antena refletora OADE, analisada pelos métodos PO, PO+PTD alimentada pela expansão de ondas esféricas e pelo MoM.

Figura 5.1 - Deslocamento no eixo de simetria para aplicação de tilt no ganho máximo.

Figura 5.2 - Diagrama de uma antena omnidirecional com o ganho máximo deslocado para $96^{\circ}$.

Figura 5.3 - Diagrama de radiação da antena refletora omnidirecional com máximo ganho descolado para $105^{\circ}$.

Figura 5.4 - Geratrizes das antenas com ganho

máximo em $96^{\circ}$ e $105^{\circ}$ comparados a parábola a $90^{\circ}$.

Figura 5.5 - Geratrizes anômala gerada pela otimização

para maximizar o ganho da antena refletora omnidirecional do caso 7_3.

Figura 5.6 - Diagrama de Radiação da análise PO+CF da otimização

para maximizar o ganho da antena refletora omnidirecional.

Figura 5.7 - Geratrizes geradas pelas otimizações para

maximizar o ganho da antena refletora omnidirecional.

Figura 5.8 - Diagrama de Radiação gerados pela análise

$\mathrm{PO}+\mathrm{CF}$ da otimização para maximizar o ganho com $\mathrm{V}_{\mathrm{S}}$ fixo.

Figura 5.9 - Geratrizes geradas pelas otimizações para

maximizar o ganho com $\mathrm{V}_{\mathrm{S}}$ fixo. 
Figura 5.10 - Geratrizes geradas pelas otimizações para

maximizar o ganho com $\mathrm{V}_{\mathrm{S}}$ fixo, com discrepâncias.

Figura 5.11 - Diagrama de Radiação gerados pela análise

$\mathrm{PO}+\mathrm{CF}$ da otimização para maximizar o ganho com $\mathrm{V}_{\mathrm{S}}$ e $\mathrm{W}_{\mathrm{A}}$ fixos.

Figura 5.12 - Superfícies geradas pelas otimizações para

maximizar o ganho com $\mathrm{V}_{\mathrm{S}}$ e $\mathrm{W}_{\mathrm{A}}$ fixos.

Figura 5.13 - Perfil de ganho dado pela função cossecante

ao quadrado com os limites.

Figura 5.14 - Diagramas de radiação gerados pela análise

(PO+CF) com 9,11 e 13 termos na Série de Fourier.

Figura 5.15 - Diagrama de radiação da Figura 5.14 em

destaque para a região de interesse na otimização.

Figura 5.16 - Superfícies modeladas com 9, 11 e 13 termos

na Série de Fourier.

Figura 5.17 - Antena refletora de duplo refletores configurada OADE.

Figura 5.18 - Diagramas de radiação, analisados por PO+CF,das

otimizações do refletor principal com 3 e 11 termos na Série de Fourier.

Figura 5.19 - (a) Geratrizes das superfícies dos refletores

principais otimizadas com 3 e 11 termos; (b) diferenças entre

a geratriz inicial e as modeladas.

Figura 5.20 - Corrente induzida sobre o refletor principal

pela fonte primária 3 e 11 termos na Série de Fourier

comparado com sem otimização.

Figura 5.21 - Diagramas de radiação analisados por PO+CF

das otimizações do subrefletor com refletores otimizados com 3 e 11 termos. 98

Figura 5.22 - (a) Geratrizes das superfícies do subrefletores

otimizados de acordo com seus refletores principais;

(b) diferenças entre o subrefletor inicial e os modelados.

Figura 5.23 - Distribuição das correntes induzidas sobre

o refletor principal após as otimizações no subrefletor. 


\section{Lista de tabelas}

Tabela 5.1 - Resultado das otimizações para família 3.

86

Tabela 5.2 - Resultado dos melhores casos das otimizações

realizadas pelas famílias 3,5,7 e 9 .

87

Tabela 5.3 - Resultado das otimizações realizadas na antena refletor com radiação omnidirecional na busca de maximizar o ganho com $\mathrm{V}_{\mathrm{S}}$ fixo.

88

Tabela 5.4- - Resultado das otimizações realizadas na antena refletor com

radiação omnidirecional na busca de maximizar o ganho com $\mathrm{V}_{\mathrm{S}}$ e $\mathrm{W}_{\mathrm{A}}$ fixos. 91

Tabela 5.5 - Configurações da OADE. 